\title{
Les débats sur la mixité des élèves dans l'enseignement privé catholique à la fin des années 1960
}

Catholic private schools and coeducation in the 1960s

Die Debatten über die Schülermischung im katholischen Privatunterricht am

Ende der 1960er Jahre

Yves Verneuil

\section{(2) OpenEdition}

Journals

Édition électronique

URL : https://journals.openedition.org/histoire-education/2609

DOI : 10.4000/histoire-education.2609

ISSN : 2102-5452

Éditeur

ENS Éditions

Édition imprimée

Date de publication : 20 octobre 2013

Pagination : $57-91$

ISBN : 978-2-84788-498-2

ISSN : 0221-6280

Référence électronique

Yves Verneuil, « Les débats sur la mixité des élèves dans l'enseignement privé catholique à la fin des années 1960 », Histoire de l'éducation [En ligne], 137 | 2013, mis en ligne le 20 octobre 2016, consulté le 20 mai 2021. URL : http://journals.openedition.org/histoire-education/2609 ; DOI : https://doi.org/ 10.4000/histoire-education.2609 


\section{Les débats sur la mixité des élèves dans l'enseignement privé catholique à la fin des années 1960}

\section{Yves VERNEUIL}

Dans les "petites écoles" de l’Ancien Régime, les garçons et les filles étaient souvent mêlés, malgré les prescriptions des évêques. La séparation des sexes n'a été vraiment mise en pratique qu'à partir du début du XIX siècle, époque d'une véritable "dégémination" selon Pierre Caspard ${ }^{1}$ que la III ${ }^{e}$ République n'a pas remis en cause dans les écoles primaires, mettant, en outre, en place un enseignement secondaire féminin séparé. Une évolution se dessine dans le premier tiers du XXe siècle : une série de circulaires autorisent, sous certaines conditions, les jeunes filles à s'inscrire dans certaines classes des lycées et collèges de garçons; un certain nombre d'écoles primaires rurales sont géminées ${ }^{2}$; une expérience de mixité est menée au lycée Marcellin Berthelot de Saint-Maur-des-Fossés ${ }^{3}$. Cependant, "l'accélération décisive " se produit dans les années 1960. Des décisions réglementaires encouragent ce mouvement. La circulaire du 3 juillet 1957 recommande la coéducation dans les établissements

1 Pierre Caspard, "À quoi tient la supériorité des filles?", Histoire de l'éducation, nº 114-115, septembre 2007, p. 136.

2 La gémination consiste à unir une école à classe unique de garçons et une école à classe unique de filles, afin d'établir une école à plusieurs classes mêlant garçons et filles.

3 Cécile Hochard, "Une expérience de mixité dans l'enseignement secondaire à la fin des années 1930 : le lycée Marcellin Berthelot à Saint-Maur-des-Fossés", Clio. Histoire, femmes et sociétés, nº 18, 2003 p. 113-124.

4 Françoise Lelièvre, Claude Lelièvre, Histoire de la scolarisation des filles, Paris, Nathan, 1991, p. 174. 
d'enseignement secondaire, non seulement pour des raisons d'économies, mais aussi dans la perspective d'une formation commune des caractères, laquelle "peut rendre les jeunes filles plus simples, plus objectives, les jeunes gens plus discrets, plus policés, tous plus compréhensifs, plus généreux". Puis, par décret du 3 août 1963, la mixité devient le régime normal des collèges d'enseignement secondaire nouvellement créés; la circulaire du 15 juin 1965 va dans le même sens pour les nouvelles écoles primaires élémentaires.

Comme l'écrit Rebecca Rogers, "si la généralisation de la mixité ne soulève guère de polémiques, il serait erroné de penser que les milieux pédagogiques n'en ont pas débattu " ${ }^{5}$, ainsi qu'en témoigne l'analyse faite par l'inspectrice générale Odette-Georges Brunschwig dans la revue L'Éducation nationale, subventionnée par le ministère ${ }^{6}$. Néanmoins la coéducation n'a pas été l'objectif principal de la mise en place de la mixité, mais plutôt la volonté de réaliser des économies (les filières se multipliant dans le second cycle du second degré, il s'agit d'éviter les doublons avec des effectifs restreints), ou bien le désir d'améliorer le dispositif pédagogique (les écoles élémentaires à cinq niveaux sont jugées désormais préférables, comme le signale la circulaire du 17 juin 1969); le but est alors de remplacer la séparation des sexes par la séparation des âges ${ }^{7}$. On peut se demander si cette évolution a également concerné l'enseignement privé. Pour la mixité comme pour d'autres sujets, l'essentiel des études concerne en effet l'enseignement public. La proportion d'élèves inscrits dans des établissements privés est pourtant loin d'être marginale : en 1970, ils sont $14 \%$ dans le primaire élémentaire ${ }^{8}$ et $21 \%$ dans le second degré ${ }^{9}$ ce qui amène à s'interroger sur la mise en place de la mixité dans l'enseignement privé et les débats qu'elle a pu y susciter. On pense volontiers que les résistances ont été plus vives dans un

5 Rebecca Rogers, "La mixité ", in François Jacquet-Francillon, Renaud d'Enfert, Laurence Loeffel (dir.), Une histoire de l'école : anthologie de l'éducation et de l'enseignement en France, XVIII -XX ${ }^{e}$ siècle, Paris, Retz, 2010, p. 178.

6 Odette-Georges Brunschwig, "L'éducation mixte», L'Éducation nationale, n 30, novembre 1961, p. 5-10.

7 Antoine Prost, Éducation, société et politique. Une histoire de l'enseignement de 1945 à nos jours, Paris, Seuil, 1992, p. 70.

8 Pour le primaire élémentaire, 671873 élèves sur 4799011 en 1970 : cf. Statistiques des enseignements, Paris, IPN, 1970-1071, p. 11 et 21.

9 Pour les établissements publics, 3218733 élèves, en comprenant ceux des lycées, des CES (collèges d'enseignement secondaire), des CEG (collèges d'enseignement général) et des CET (collèges d'enseignement technique); pour l'enseignement privé, 1262775 élèves, en comprenant ceux des établissements de niveau lycée, ceux des établissements de niveau lycée technique, ceux des cours complémentaires et ceux des centres d'apprentissage. Cf. Statistiques des enseignements, Paris, IPN, 1969-1970. 
monde où $95 \%$ des établissements sont catholiques et où ne s'était pas perdu le souvenir de l'encyclique pontificale Divini illius Magistri (1929), qui prohibait la coéducation. Mais faut-il s'en tenir à suggérer les réticences de l'enseignement catholique? En fait, des débats ont existé qui demeurent méconnus, si bien que l'on en reste le plus souvent, pour l'enseignement privé, au domaine des représentations, alors même que, dans les années 1960, le monde catholique est lui aussi en pleine évolution, après le concile de Vatican II. Il convient par conséquent de chercher d'abord à prendre la mesure statistique du phénomène. En utilisant périodiques et publications issus des milieux éducatifs catholiques, on montrera ensuite la diversité des positions concernant la mixité dans les établissements scolaires catholiques, et on se demandera s'il existe une position officielle formulée par le Secrétariat général de l'enseignement catholique ${ }^{10}$. On cherchera enfin à comprendre ce que recouvre, pour l'enseignement catholique, la coéducation en matière de relations entre les sexes.

\section{Un phénomène croissant}

\section{Une forte progression dans le primaire élémentaire}

Dans l'enseignement privé, comme dans l'enseignement public, les écoles maternelles ont toujours été mixtes. Comme le souligne le $\mathrm{D}^{\mathrm{r}} \mathrm{Le}$ Moal, lors d'une conférence donnée à l'Institut catholique de Paris, le 17 décembre 1967 dans le cadre du Centre catholique d'éducation familiale, à l'école maternelle la mixité n’a jamais posé de problème, "car on vivait avec l'idée que les petits étaient asexués. L'hostilité à l'égard de la mixité s'est manifestée surtout à propos des classes primaires et plus encore des classes secondaires, en raison de ce que l'on considérait comme l'éveil de la sexualité "11.

Pour ce qui concerne l'enseignement primaire élémentaire, les statistiques montrent une nette progression de la proportion des écoles privées mixtes,

10 Le secrétaire général de l'enseignement privé n'est pas le "chef» de l'enseignement privé; il a un simple rôle d'influence et d'impulsion. Lui-même est nommé par l'épiscopat; ses adjoints sont désignés par la commission permanente du Comité national de l'enseignement catholique (CNEC). Depuis 1965, les membres de cet organisme, dont le pouvoir est essentiellement moral, sont nommés par les organisations représentatives de l'enseignement privé, et non plus par les évêques (Edmond Vandermeersch, École : Église et laïcité. Souvenirs autour de la loi Debré (1960-1970), Paris, L’Harmattan, 2008, p.61-66).

11 Centre catholique d'éducation familiale ACGF [Action catholique générale des hommes] et ACGH [Action catholique générale féminine), Mixité et coéducation, Paris, Centre catholique d'éducation familiale, 1968, p. 16. Le $\mathrm{D}^{\mathrm{r}}$ Le Moal est directeur technique du Centre d'observation des mineures délinquantes à Chevilly-Larue. 
même si l'enseignement privé accuse un retard important par rapport à l'enseignement public. Ce retard explique la plus forte progression de la mixité dans les établissements primaires privés : alors qu'elle n'était que de $12 \%$ en 1963-1964, elle atteint presque la moitié des établissements en 1970-1971. La mutation est donc rapide, comme le montre le tableau 1 :

\begin{tabular}{|l|c|c|c|c|c|c|c|c|}
\hline \multirow{2}{*}{ Années } & \multicolumn{4}{|c|}{ Enseignement public } & \multicolumn{4}{c|}{ Enseignement privé } \\
\cline { 2 - 9 } & G & F & mixte & $\%$ & G & F & mixte & $\%$ \\
\hline $1963-1964$ & 13162 & 13181 & 38124 & $59 \%$ & 2809 & 5027 & 1441 & $12 \%$ \\
\hline $1964-1965$ & 12500 & 12482 & 38814 & $61 \%$ & 2726 & 4838 & 1628 & $18 \%$ \\
\hline $1968-1969$ & 7383 & 7428 & 24252 & $62 \%$ & 1963 & 3137 & 2048 & $29 \%$ \\
\hline $1970-1971$ & 5269 & 5290 & 26794 & $72 \%$ & 1439 & 2092 & 3167 & $47 \%$ \\
\hline
\end{tabular}

Tableau 1 : la mixité dans les écoles primaires élémentaires publiques et privées, 1963-1971 ${ }^{12}$. $\mathrm{G}=$ écoles spéciales de garçons; $\mathrm{F}=$ écoles spéciales de filles; mixte = écoles mixtes ; $\%=$ pourcentage d'écoles mixtes

Dans l'enseignement privé, les écoles spéciales de filles sont plus nombreuses que celles de garçons, alors que les nombres sont à peu près similaires dans l'enseignement public. Il est vrai que dans l'enseignement privé, les filles restent plus nombreuses que les garçons, alors que c'est le contraire dans l'enseignement primaire public (tableau 2). Ces données correspondent à un héritage : les milieux catholiques ont longtemps privilégié les écoles de filles; par ailleurs, des parents qui mettaient leurs garçons dans une école publique mettaient parfois leurs filles dans une école privée, pour des raisons d'éducation morale.

\begin{tabular}{|c|c|c|c|c|c|c|}
\hline \multirow{2}{*}{ années } & \multicolumn{3}{|c|}{ Enseignement public } & \multicolumn{3}{c|}{ Enseignement privé } \\
\cline { 2 - 7 } & garçons & filles & total & garçons & filles & total \\
\hline $1963-64$ & 2438792 & 2260359 & 4699151 & 375011 & 473957 & 848968 \\
\hline $1970-71$ & 2136685 & 1990453 & 4127138 & 313481 & 358392 & 671873 \\
\hline
\end{tabular}

Tableau 2 : effectifs des garçons et des filles dans les écoles primaires élémentaires publiques et privées, $1963-1971^{13}$.

Dans le premier tiers du $\mathrm{XX}^{\mathrm{e}}$ siècle, les milieux cléricaux condamnaient la politique de gémination d'écoles rurales pratiquée par les pouvoirs publics

12 Sources : Annuaire statistique de la France, 1965, p. 102; Annuaire statistique de la France, 1967, p. 121 ; Statistiques des enseignements, Paris, IPN, 1968-1969, p. 3 et 6; ibid., 1970-1971, p. 5 et 9.

13 Annuaire statistique de la France, 1965, p. 103; Statistiques des enseignements, Paris, IPN, 19701971, p. 11 et 21 . 
dans le but de remplacer les écoles à classe unique par des écoles géminées à deux classes, dans la perspective d'une meilleure efficacité pédagogique. Mais en pratique, l'Église catholique avait toléré le développement d'écoles rurales mixtes. Alors que les écoles privées étaient majoritairement des écoles féminines, cette politique d'accommodement avait pour vertu de remplir les petites écoles rurales et de ne pas rejeter les garçons vers l'enseignement public. Sara Teinturier a ainsi pu montrer que la mixité s'est développée en Bretagne dans les écoles privées dès les années $1930^{14}$. Dans les années 1960, l'enseignement public accentue sa mutation, qui va progressivement substituer la séparation des âges à la séparation des sexes. Antoine Prost a insisté sur cette mutation fondamentale ${ }^{15}$. L'enseignement privé suit le mouvement : à son tour, il pratique la gémination d'écoles, et tend à supprimer, quand c'est possible, les écoles à classe unique pour les remplacer par des écoles géminées, dans lesquelles garçons et filles sont réunis, les plus jeunes étant dans l'une, les plus âgés dans l'autre. Il s'agit de pallier le manque d'effectifs dans les petites écoles rurales, mais aussi d'améliorer l'efficacité pédagogique, les écoles à cinq classes, du $\mathrm{CP}$ jusqu'au CM2, étant réputées de meilleur niveau : or la poursuite d'études dans le second degré se généralise et, dans le cadre des Trente Glorieuses, les parents sont soucieux d'élévation sociale par l'acquisition de diplômes. En 1966, comme le montre le tableau 3, l'enseignement privé, contrairement à l'enseignement public, compte même davantage d'écoles mixtes à plusieurs classes que d'écoles mixtes à classe unique (la proportion d'écoles mixtes est supérieure dans l'enseignement public, mais celui-ci doit aussi faire aux contraintes du service public et maintenir des écoles dans les petites communes rurales) :

\begin{tabular}{|l|r|r|c|}
\hline & public & \multicolumn{1}{|c|}{ privé } & \multicolumn{1}{c|}{ total } \\
\hline Écoles mixtes à classe unique & 20737 & 479 & 21216 \\
\hline Écoles mixtes à plusieurs classes & 18017 & 1149 & 19166 \\
\hline Écoles de garçons & 12500 & 2726 & 15226 \\
\hline Écoles de filles & 12482 & 4838 & 17320 \\
\hline
\end{tabular}

Tableau 3 : nombre d'écoles primaires élémentaires en $1966^{16}$.

14 Sara Teinturier, L'Enseignement privé dans l'entre-deux-guerres. Socio-histoire d'une mobilisation catholique, thèse de doctorat, université de Rennes II, 2013.

15 Antoine Prost, Histoire de l'enseignement et de l'éducation, t. 4 : Depuis 1930, Paris, Perrin, 2004, p. 172-173.

16 Tableaux de l'Éducation nationale, 1966, p. 30. 
La politique de gémination permet en outre de pallier le manque de directeurs hommes, car les écoles géminées peuvent avoir chacune une directrice (selon la loi du 30 octobre 1886, les écoles privées mixtes doivent, comme les écoles publiques, être dirigées par des institutrices ${ }^{17}$ ). Le 8 avril 1970, l'inspecteur d'académie d'Ille-et-Vilaine transmet ainsi une demande de direction unique pour les écoles privées géminées de Saint-Seglin. Il précise que la demande est essentiellement motivée par la difficulté qu'éprouve l'autorité à recruter du personnel masculin, réglementairement qualifié, pour assurer la direction des écoles sous contrat ${ }^{18}$.

\section{Une progression dans l'enseignement secondaire}

On dispose de statistiques sur la mixité dans les établissements secondaires publics, mais pas dans l'enseignement secondaire privé. On sait juste que, comme dans le primaire, les filles y sont plus nombreuses : en 1964-1965, on compte dans les établissements secondaires classiques et modernes du privé 1,08 filles pour 1 garçon $^{19}$. Ces chiffres témoignent d'ailleurs de la bonne poursuite d'études des filles, moins nombreuses que les garçons dans le primaire. On ignore toutefois si les jeunes filles sont davantage scolarisées dans des établissements mixtes ou dans des établissements spécifiques, si bien que l'on en est réduit aux conjectures. D’après la revue Échanges, publiée par les sœurs auxiliatrices, la moitié des classes de terminale seraient mixtes en $1966^{20}$; mais sur quoi se base cette estimation? Par ailleurs, selon Antoine Prost, dans les années 1960, "seuls certains établissements privés - les plus chics - continuent à recruter des élèves d'un seul sexe ${ }^{21}$. Les "terres de chrétienté» ont pu toutefois respecter plus longtemps la tradition de la séparation des sexes. Ainsi, à Coutances, capitale épiscopale du Cotentin et terrain de "guerre scolaire",

17 Le conseil départemental peut cependant autoriser un instituteur privé à diriger une école mixte, à la condition qu'il lui soit adjoint une maîtresse de couture. Cf. "École privée", in Ferdinand Buisson (dir.), Nouveau dictionnaire de pédagogie et d'instruction primaire, Paris, Hachette, 1911.

18 Archives nationales [désormais AN], 19930639/1.

19 Annuaire statistique de la France, 1967, p. 128. Dans les établissements publics équivalents, on compte 1,05 filles pour 1 garçon.

20 Marc Harteman, "Coéducation des filles et des garçons dans l'enseignement", Échanges, n 76 , Pentecôte 1966, p. 16. La rédactrice en chef de la revue Échanges est la sœur Françoise Vandermeersch, sœur d'Edmond Vandermeersch, secrétaire général adjoint de l'Enseignement catholique, avec qui elle partage des idées progressistes (cf. Sabine Rousseau, Françoise Vandermeersch. L'émancipation d'une religieuse, Paris, Karthala, 2012).

21 Antoine Prost, Histoire de l'enseignement et de l'éducation, op. cit, p. 579. 
le primaire et le premier cycle du second degré ne sont devenus mixtes qu'au milieu des années $1980^{22}$. En revanche, le second cycle du second degré (le lycée privé Germain) s'était ouvert aux filles dès la fin des années 1960, par la force des choses, car il n'existait pas de lycée privé de jeunes filles ${ }^{23}$.

Si à Coutances, l'introduction de la mixité a été particulièrement tardive, les modalités de son introduction n'y ont pas été originales : alors même que l'on pourrait penser que la mixité présente davantage de risques pour les 15-18 ans que pour les 11-15 ans, elle a, le plus souvent, été mise en place dans le second cycle du second degré avant de l'être dans le premier cycle. Ce sont en effet les classes terminales qui ont été les premières concernées, un peu comme elles l'avaient été dans les lycées publics de garçons dès les années 1920. Pour proposer aux parents le plus grand nombre de filières et d'options possibles, et avoir dans chacune des effectifs suffisants, les établissements ont été amenés à accueillir des enfants des deux sexes. Cela peut conduire à une spécialisation des établissements, en particulier dans les moyennes et grandes villes. Plusieurs établissements s'accordent pour proposer ensemble le choix d'options le plus large possible. Ce fut le cas à Troyes : instaurée au début des années 1970 au terme d'un accord entre le lycée de filles Saint-François-de-Sales et le lycée de garçons Saint-Bernard (un établissement offre les terminales B, l'autre les terminales C), la mixité des classes terminales a précédé d'une quinzaine d'années la mixité dans les classes du premier cycle du second degrée ${ }^{24}$. Cette modalité de mise en place de la mixité est la plus courante à la fin des années 1950, et jusqu'au milieu des années 1960. Si les établissements parisiens les plus prestigieux y échappent, comme SaintLouis-de-Gonzague, dans le $16^{\mathrm{e}}$ arrondissement de Paris, qui n'adopte la mixité qu'en 1980, ce n'est pas tant que les parents des "beaux quartiers" soient plus réticents envers la mixité, que parce que ces établissements n'ont pas de problème d'effectifs et donc pas besoin de recruter des enfants de l'autre sexe. Leur situation est très différente de celles des petites et moyennes villes de province, où l'on a besoin de remplir les classes terminales. Alors que le climat est réputé plus ouvert dans les grandes villes que dans les petites villes de province, la mixité

22 Dans le primaire, les élèves du cours élémentaire vont dans l'ancienne école de filles, les élèves du cours moyen dans l'ancienne école de garçons. Cette pratique, habituelle dans les cas de gémination, reflète les stéréotypes de genre, qui considèrent qu'une maitresse est mieux appropriée pour les petits. Elle n'a pas été spécifique à l'enseignement privé.

23 Témoignage du 30 mars 2013.

24 Témoignage du 15 janvier 2013. 
y progresse moins vite, ce que souligne le père mariste Rey-Herme, professeur à l'Institut supérieur de pédagogie (ISP) de Paris, lui-même favorable à la mixité, lors d'une conférence organisée par sa congrégation en mars 1968 : "À Paris, par exemple, où les élèves sont assez nombreux dans chaque classe, la proportion des classes mixtes est très inférieure à celle de la province. Ce qui signifie que la décision d'un grand nombre d'établissements scolaires privés de pratiquer la mixité a été prise sous la pression de nécessités financières ${ }^{25}$.

Ce n'est que dans un second temps, à partir de la fin des années 1960 et surtout dans les années 1970, que l'introduction de la mixité se veut liée à une démarche éducative. On estime alors qu'il vaut mieux habituer précocement les enfants des deux sexes à se côtoyer, si bien que la mixité est introduite à partir des classes du premier cycle, en allant vers les classes terminales, année par année. Cette solution permet de prolonger la mixité qui est de plus en plus courante dans les écoles primaires. C'est cette manière de faire qu'adopte Antoinette Salmon-Legagneur, au début des années 1970, pour l'établissement parisien de jeunes filles qu'elle dirige dans le $10^{\mathrm{e}}$ arrondissement (Saint-Vincent de Paul, rue de Rocroy). Elle s'appuie sur la mixité des classes primaires de l'établissement pour l'introduire progressivement à partir de la classe de sixième ${ }^{26}$. Dans l'école-collège Notre-Dame à Reims, vieil établissement féminin dont les origines remontent au XVII ${ }^{\mathrm{e}}$ siècle, la direction, qui réfléchit à la fin des années 1970 au passage à la mixité, a cependant envisagé de l'établir seulement au collège. Le but est éducatif (l'établissement, pas plus que les autres établissements privés de Reims, n'a de souci d'effectifs ${ }^{27}$ ) : il s'agit de préparer les élèves au monde dans lequel ils seront appelés à vivre, et la cohabitation avec l'autre sexe apparaît importante à l'âge de l'adolescence. Après deux années de réflexion de l'équipe éducative, en concertation avec les parents d'élèves (très influents dans l'enseignement privé), il est toutefois décidé que l'école primaire sera elle aussi mixte. C'est donc l'ensemble scolaire (excepté le pensionnat) qui passe à la mixité en 1979.

25 Mixité et coéducation, Dossier CDM, n85, Centre de documentation mariste, mars 1969, p. 3.

26 Antoinette Salmon-Legagneur, témoignage du 20 janvier 2011.

27 C'était plutôt l'inverse. Aussi, dans le souci que tous les élèves puissent être accueillis sur Reims, la direction de l'établissement Notre-Dame a sollicité le pensionnat du Sacré-Cœur et le lycée SaintJoseph afin de réfléchir en même temps au passage à la mixité. Le premier accepte, mais diffère sa décision de deux ans; le second refuse. L'établissement dirigé par les jésuites tient alors à poursuivre sa tradition d'enseignement masculin. Il ne passe à la mixité qu'en 1993 : du fait de la baisse des effectifs, les résistances cèdent alors le pas devant les nécessités économiques. La mixité commence en classe de CP, puis, année après année, atteint les différentes classes du collège, puis du lycée. 
L'introduction de la mixité à partir de la classe de sixième n'est pas toujours, toutefois, liée à un idéal coéducatif. Au lycée de La Providence d'Amiens, la question était plutôt celle des effectifs. Dans le but d'accroître le nombre de classes secondaires, l'établissement dirigé par les jésuites avait abandonné, au cours des années 1960, ses classes primaires. Mais les établissements primaires privés avec lesquels il avait passé un accord ont ouvert par la suite des classes secondaires, à la demande des familles. La Providence en subissait les conséquences de plein fouet : en 1973-1974, date à laquelle est prise la décision d'introduire la mixité, il n'y avait plus que quarante élèves en sixième, ce qui compromettait la prospérité, voire l'existence de l'établissement; des enseignants étaient sur le point de perdre leur emploi, suite à des fermetures de classes. La direction décide alors d'introduire la mixité, en commençant par la sixième puisque c'est dans cette classe que la situation était la plus critique ${ }^{28}$.

$\mathrm{Au}$ total, il n'est pas certain qu'il soit possible d'établir de différenciations régionales et que l'on puisse opposer, par exemple, "terres de chrétienté" et terres précocement déchristianisées. Certes, on peut trouver d'autres exemples d'établissement tardif de la mixité dans des régions catholiques : ainsi le lycée Saint-Joseph de Besançon n'est-il devenu mixte qu'en 1989, à l'occasion de sa fusion avec un autre établissement, Sainte-Ursule. Mais il est aussi des mixités précoces : le lycée Saint-Martin de Rennes, tenu par les pères eudistes, l'adopte dès les années 1960; de même, le collège de l'Adoration, dans la même ville, passe à la mixité en 1968. Inversement, à Troyes, dans une région déchristianisée, l'école-collège-lycée Saint-François, tenu par les sœurs oblates de Saint-François de Sales, a longtemps résisté à la mixité : acceptée pour le CP dès 1968, elle n'est établie qu'en 1987 pour le reste de l'école primaire, en 1988 pour le lycée et en 1989 pour le collège. La mixité est établie en dernier au collège, car l'âge des élèves du collège est considéré comme sensible. Cependant, les parents la demandent, de manière à pouvoir confier la scolarité complète de leur garçon à l'établissement, du primaire au lycée. Aussi la directrice, sœur Geneviève Poinsot, s'y résout-elle. Elle-même, qui avait fait sa scolarité dans un collège public ouvert aux deux sexes, mais ayant des classes de garçons et des classes de filles ${ }^{29}$,

28 Témoignage du père Dominique Bouzy, 22 avril 2014.

29 Il s'agit du collège de Bar-sur-Seine, où Geneviève Poinsot était élève entre 1959 et 1963 . En cas de manque d'effectifs, quelques garçons étaient inscrits dans les classes de filles ou quelques filles dans les classes de garçons, mais dans la mesure du possible la direction du collège cherchait à avoir des classes non mixtes (témoignage de sœur Geneviève Poinsot, mercredi 12 mars 2014). 
était réticente. Le caractère tardif de la mixité était toutefois une décision des mères supérieures de la congrégation, qui tenaient à préserver la tradition d'un enseignement féminin. La politique des congrégations, le contexte géographique et social propre à chaque établissement, la concurrence avec d'autres établissements, peuvent donc déterminer des politiques différentes d'un établissement à un autre, dans un même lieu ou un même département. Ce n'est guère étonnant, si l'on considère qu'une des spécificités de l'enseignement privé réside justement dans l'originalité de chaque établissement, en particulier dans l'enseignement secondaire.

\section{II - Évolutions sociales et nouveaux modèles pédagogiques}

\section{1 - L'évolution des mentalités}

Comme l'a montré Anne-Marie Sohn, les mentalités évoluent dans les années 1950 et 1960 au sujet des relations entre les sexes : jeunes gens et jeunes filles se voient au cinéma, en vacances ${ }^{30}$. Le flirt des adolescents reste cependant considéré comme une menace par bien des parents et les conceptions de ceux qui mettent leurs enfants dans les établissements catholiques sont certainement plus conservatrices que la moyenne. Par ailleurs, comme le remarque à cette époque Edmond Vandermeerch, alors directeur du collège Saint-Joseph à Lille, ce sont parfois les mêmes parents qui acceptent que les enfants organisent chez eux des fêtes réunissant des adolescents des deux sexes, mais qui demeurent réticents à la mixité scolaire ${ }^{31}$. Au collège de l'Assomption de Briey (Meurthe-et-Moselle), qui a pourtant adopté précocement pour la mixité au milieu des années 1950, lorsque l'on entrevoit le début d'un flirt ou d'un sentiment amoureux entre deux élèves, on n’hésite pas à prendre des mesures radicales : «Une séparation s’avère parfois nécessaire, estime le directeur. Elle n'a rien d'infamant. On nous cite l'exemple d'élèves qui ont terminé leurs études dans des collèges distincts et qui se sont retrouvés après pour former un foyer heureux" ${ }^{32}$.

La question des flirts est donc au cœur de la controverse qui oppose partisans et adversaires de la mixité. Ces derniers s'en font les Cassandre : on verra

30 Anne-Marie Sohn, Age tendre et tête de bois. Histoire des jeunes des années 1960, Paris, Hachette, 2001 ; et "Les "relations filles-garçons" : du chaperonnage à la mixité (1870-1970)", Travail, genre, sociétés, nº 9, 2003/1, p.91-109.

31 Témoignage d'Edmond Vandermeersch, 10 mars 2011.

32 Famille éducatrice, $\mathrm{n}^{\circ} 10$, décembre 1966, p.3. 
se multiplier le nombre des élèves tombées enceintes. À l'inverse, dans la revue Échanges, le jésuite Marc Harteman estime que, de toutes façons, les flirts ont plutôt lieu en dehors de l'école, où la vie est mixte. Et à ceux qui pointent du doigt le risque de "défloraison psychologique", il répond que les contacts avec l'autre sexe serviront plutôt à "éviter les désillusions futures" " $^{33}$. De même, lors d'une conférence organisée sous l'égide de sa congrégation, le père mariste Rey-Herme, professeur à l'ISP, fait valoir que la mixité peut réduire le nombre des divorces, à une époque où l'on déplore qu'ils soient en augmentation : "Il faudrait tout de même s'entendre : ce n'est pas en éduquant les garçons d'un côté et les filles de l'autre qu'on peut réaliser la préparation nécessaire au mariage; ce n'est pas non plus grâce à huit jours de session pour fiancés qu'on va apprendre à connaître son propre développement affectif et le développement affectif de l'autre sexe " ${ }^{34}$. Pour sa part, lors de sa conférence donnée à l'Institut catholique de Paris, le $\mathrm{D}^{r}$ Le Moal, après avoir estimé que la coéducation supprime les curiosités malsaines, non seulement fait valoir que les établissements unisexués ne préservent pas toujours la morale, mais encore assure qu'on y rencontre plus souvent des cas d'homosexualité ${ }^{35}$. Faisant référence à l'encyclique Sacra Virginitas du 25 mars 1954, qui demandait aux éducateurs d'inculquer aux adolescents les principes de la pudeur chrétienne, le mensuel Défense du foyer s'interroge: "Si la mixité se développe avec la camaraderie "filles-garçons", y aura-t-il encore des vocations? „" . La mixité est en effet soupçonnée de conduire à tarir le recrutement des prêtres. Ce point ne peut manquer d'inquiéter les catholiques. Aussi, l'article de la Famille éducatrice qui fait l'apologie de la mixité demande-t-il son avis à l'aumônier lui-même : celui-ci admet que l'établissement dans lequel il exerce n'a suscité aucune vocation depuis l'instauration de la mixité; il estime toutefois que cela n'a rien à voir avec cette dernière, "mais au climat sociologique et à l'époque dans laquelle nous vivons ${ }^{37}$. De même, Marc Harteman, dans la revue Échanges, se dit convaincu que la mixité ne détruira pas les vocations à entrer dans les ordres; seulement, "les vocations que l'école mixte découragera seront compensées par la qualité de celles qu'on y verra germer ${ }^{38}$.

33 Marc Harteman, art. cit., p. 18.

34 Mixité et coéducation, Dossier CDM, n85, Centre de documentation mariste, mars 1969, p. 11.

35 Centre catholique d'éducation familiale ACGF et ACGH, Mixité et coéducation, op. cit.

36 Défense du foyer, $\mathrm{n}^{\circ} 84$, février 1967, p. 7.

37 Famille éducatrice, $\mathrm{n}^{\circ} 10$, décembre 1966, p.3.

38 Marc Harteman, art. cit., p. 19. 
L'exemple des mouvements de jeunesse a-t-il joué un rôle? Comme l'écrit Antoine Prost, dès les années 1950, "peu à peu, la mixité [y] progresse" En 1968, pour introduire la conférence du $\mathrm{D}^{\mathrm{r}}$ Le Moal à l'Institut catholique de Paris, M. Jean, professeur dans un établissement mixte, souligne d'ailleurs que les camps de vacances, les voyages organisés et les journées de récollection sont de plus en plus souvent mixtes ${ }^{40}$. C'est également le cas de la Jeunesse agricole catholique (JAC), qui, en 1965, s'ouvre aux deux sexes et se transforme en Mouvement rural de jeunesse chrétienne (MRJC). Cependant, la Jeunesse ouvrière chrétienne (JOC) et la Jeunesse ouvrière chrétienne féminine (JOCF) restent séparées jusqu'en 1987. Cela explique peut-être qu'Anthony Favier puisse écrire : "Je ne crois pas que les mouvements de jeunesse catholique, qu'ils soient d'Action catholique spécialisée ou scouts, aient été des éléments moteurs du changement social sur la question de la mixité dans les années soixante et soixante-dix ${ }^{41}$. Ce spécialiste de la JOC signale cependant que la question de la mixité est un sujet de débat à la fin des années 1950 et dans les années 1960. Il en va de même dans le mouvement scout. Ainsi, en 1967, dans la Belgique voisine, les autorités de la Fédération des scouts catholiques disent reconnaître que "le temps n'est plus aux discussions, la mixité est un état de fait $"^{42}$. Selon Thierry Scaillet, qui a étudié cet exemple, la demande provient des parents eux-mêmes : les contacts entre filles et garçons sont peu à peu considérés comme indispensables en matière éducative, afin de s'habituer à réfléchir aux attitudes des personnes des deux sexes; cela permettrait en outre aux scouts de trouver leur future épouse dans un "lieu sûr" ${ }^{43}$. Cependant, en France, les mouvements scouts catholiques, contrairement aux autres ${ }^{44}$, ne procèdent pas à l'unification entre organisations masculines et organisations féminines : Scouts

39 Antoine Prost, Histoire de l'enseignement et de l'éducation, op. cit., p. 578.

40 Centre catholique d'éducation familiale ACGF et ACGH, Mixité et coéducation, op. cit.

41 Anthony Favier, "L'enjeu de la mixité dans un autre mouvement de jeunesse catholique : JOC et JOCF. Une approche historique", Archives ouvertes, 8 mars $2012:<<$ http://halshs.archives-ouvertes.fr/halshs-00731504>.

42 Cité par Thierry Scaillet, "L'élément féminin au sein de la Fédération des scouts catholiques, 1929-1979", in Thierry Scaillet, Sophie Wittemans, Françoise Rosart (dir.), Guidisme, scoutisme et coéducation : pour une histoire de la mixité dans les mouvements de jeunesse, Louvain-la-Neuve, Bruylant-Academia, 2007, p. 112. En Belgique, l'épiscopat accepte cette évolution et envoie même des directives en ce sens (ibid., p. 125).

43 Ibid., p. 106.

44 En 1950, les Éclaireurs de France s'ouvrent aux filles; en 1964, la Fédération française des éclaireuses se dissout et ses sections fusionnent avec leur équivalent masculin. 
de France et Guides de France demeurent séparés. Signe néanmoins que les mentalités évoluent : une tentative apparait, avec l'institution des "Jeunes en marche" (JEM). Cette création s'adresse aux jeunes gens et aux jeunes filles de plus de 17 ans, avec l'idée qu'ils sont souvent appelés à pratiquer la mixité à l'université ou... au lycée. Dès 1968, les Scouts de France s'éloignent de ce projet, mais il semble que ce soit essentiellement pour des raisons d'approche différente de la pédagogie scoute. Il reste que, en 1973, Scouts de France et Guides de France réaffirment comme une orientation commune la nécessité de rechercher les conditions qui permettent aux membres des deux mouvements de "tirer un enrichissement d'une situation de mixité et les préparer ainsi à être mieux hommes et femmes dans la société où nous vivons. Pour cela, développer une attitude positive vis-à-vis de la mixité et, en attendant, assurer les situations qui existent sur le terrain $"^{45}$.

Même s'il faut nuancer l'influence des mouvements de jeunesse catholiques, qui se disent eux-mêmes influencés par la mixité scolaire, il est clair, néanmoins, que les parents se rendent bien compte de la généralisation de la mixité en dehors de la vie scolaire; Antoine Prost le rappelle : c'est "le temps des copains " ${ }^{46}$. Assurément, les mentalités évoluent moins vite chez les catholiques. À travers l'exemple de l'Union féminine civique et sociale (UFCS), de tradition catholique ${ }^{47}$, Mathilde Dubesset a montré à quel point le thème de la maternité et de la femme au foyer reste présent dans les années 1960. Mais elle souligne également que, dans ces années 1960 où tout bouge, les catholiques aussi ont conscience qu'est progressivement mis à distance le modèle de la femme assignée à son intérieur, dont la destinée serait circonscrite à la maternité ${ }^{48}$. De même, Sylvie Chaperon a rappelé qu'après être restée en retrait sur la question du travail féminin, et avoir considéré que la mère a des devoirs prioritaires envers son enfant, l'UFCS a évolué, comme le montre son congrès national de 1964 organisé autour du thème "Dans un monde en évolution :

45 Cité par Marie-Thérèse Cheroutre, Le Scoutisme au féminin : les Guides de France, 1923-1998, Paris, Cerf, 2002, p. 375.

46 Antoine Prost, Histoire de l'enseignement et de l'éducation, op. cit., p. 578-580.

47 En 1965, l'UFCS se déconfessionnalise (Mathilde Dubesset, "Un féminisme chrétien à gauche?», in Denis Pelletier, Jean-Louis Schlegel (dir.), À la gauche du Christ. Les chrétiens de gauche en France de 1954 nos jours, Paris, Seuil, 2012, p. 402).

48 Mathilde Dubesset, "Les figures du féminin à travers deux revues féminines, l'une catholique, l'autre protestante, La femme dans la vie sociale et Jeunes femmes, dans les années 1950-1960", Le Mouvement social, n 198, 2002/1, p. 29. 
la femme et son travail "49. Témoignage de l'évolution des esprits, le mensuel Panorama chrétien croit pouvoir écrire, avec d'ailleurs une pointe d'optimisme :

"La mixité n'est d'ailleurs pas seulement un phénomène scolaire. C'est un phénomène social d'abord, un fait étroitement lié à l'évolution de la femme moderne. À l'âge adulte, les milieux professionnels sont tous devenus mixtes et tendent chaque jour à le devenir à égalité de compétence et de responsabilité (sinon de rémunération). Si l'on songe au nombre d'heures de vie professionnelle et à l'importance qu'elles revêtent à l'âge adulte, on peut dire que la mixité est devenue une constance pour les hommes et les femmes de notre temps (et ceci est vrai, aussi, pour les jeunes adultes que sont nos étudiants de faculté). Si l'on songe que la vie familiale (basée d'abord sur le couple) et la vie de loisir (du moins ceux qui ne sont pas encadrés et dirigés) sont elles aussi mixtes, le domaine de la non-mixité se réduit finalement à peu de choses : les seules activités scolaires ${ }^{50}$.

Assurément, ces considérations négligent les pesanteurs et les inégalités d'emplois entre hommes et femmes : comme le note Christine Bard, "la féminisation des professions qualifiées date surtout des années 1980 „51. Par ailleurs, subsiste dans les milieux chrétiens conservateurs l'idéal de la femme au foyer et d'une stricte séparation des rôles masculins et féminins. En témoigne cet extrait d'un article de Défense du foyer :

"La fille est faite pour devenir une femme avec toute sa délicatesse, sa finesse, sa sensibilité : pour devenir, avec toute sa capacité d'aimer, une épouse et une mère. Le garçon est fait pour devenir un homme avec toutes les qualités de virilité, de dévouement, de travail et d'autorité, que réclameront [sic] plus tard son triple titre d'époux, de père et de membre de la cité ${ }^{52}$.

L'article de Panorama chrétien montre néanmoins que ce discours, qui n'est pas sans rappeler la valorisation de l'"éternel féminin " à l'honneur à l'époque du régime de Vichy ${ }^{53}$, n'est plus accepté par tous les catholiques. En outre, même dans les familles bourgeoises catholiques, on désire de plus en plus que les filles fassent des études "sérieuses", débouchant sur le baccalauréat (même si ces familles sont souvent moins ambitieuses pour les filles et ne les orientent pas

49 Sylvie Chaperon, Les Années Beauvoir : 1945-1970, Paris, Fayard, 2000, p. 289.

50 Jeanne-Marie Fauré, "Nos garçons et nos filles à l'école mixte", Panorama chrétien, nº 126, septembre 1967, p.66. La revue Panorama chrétien regroupe des chrétiens progressistes du milieu rural, proches de la JAC.

51 Christine Bard, Les Femmes dans la société française au XXe siècle, Paris, Armand Colin, 2001, p. 217.

52 Défense du foyer, février 1967, p. 8.

53 Francine Muel-Dreyfus, Vichy et l'éternel féminin, Paris, Seuil, 1996. 
forcément vers les mêmes voies). De la part des parents, il ne s'agit pas forcément d'envisager une profession; souvent les études sont plutôt une précaution. Néanmoins, Edmond Vandermeersch observait, dans les établissements réputés de Lille, qu'il n'était pas rare que des jeunes filles ayant fait leurs études dans des établissements catholiques deviennent institutrices dans des établissements publics. Ce mouvement se traduit par la féminisation de l'enseignement secondaire privé : c'est en 1949-1950 que le nombre d'élèves filles dépasse pour la première fois le nombre d'élèves garçons dans le second degré privé ${ }^{54}$.

$\mathrm{Au}$ reste, l'image de la jeune fille faisant des études a changé, comme en témoigne ce nouveau lieu commun voyant dans la "coéducation des sexes" un facteur favorable à l'émulation. Ce topos constitue un vrai renversement par rapport à l'époque où l'on considérait que l'activité intellectuelle de la jeune fille risquait de la dénaturer et de la transformer en femme savante ${ }^{55}$. L'égalité intellectuelle entre les sexes est davantage admise, ce qui ne veut pas dire qu'elle l'est complètement, comme en témoignent les différences d'orientation scolaire et professionnelle. Henry Moreau, qui a mené avec Antoine de La Garanderie, délégué national de l'UNAPEL, une enquête sur la mixité, écrit ainsi dans le mensuel Famille éducatrice :

"Les garçons ne doivent pas se targuer d'une supériorité intellectuelle tenant à la nature de leur sexe. Mais pour qu'ils s'en rendent compte, il faut qu'ils aient l'occasion de s'en apercevoir. Inversement, les filles pourront d'autant mieux affirmer leur personnalité intellectuelle qu'elles ne sentiront pas le poids d'une appréciation masculine qui les infériorise $"^{56}$.

Ce désir de faciliter les études des filles favorise, en province, l'instauration de la mixité dans des collèges de garçons, pour des raisons de commodité. Le directeur du collège de l'Assomption de Briey rapporte ainsi que "ce sont les parents eux-mêmes qui l'ont demandée, cette mixité. Ils devaient envoyer leurs filles en pension. Metz est à $30 \mathrm{~km}$, Longwy à 50 , Nancy à 80 . Briotins, ils étaient défavorisés. Alors, ils envoyèrent une délégation à l'évêché, et $\mathrm{M}^{\mathrm{gr}}$ Lallier en référa à la Commission épiscopale en 1954. Nos débuts furent très prudents. On admit d'abord les filles en sixième et en cinquième. Et c'est ainsi qu'en 1957

54 Annuaire statistique de la France, 1966, p. 142.

55 Nicole Mosconi, "La femme savante. Figure de l'idéologie sexiste dans l'histoire de l'éducation", Revue française de pédagogie, $\mathrm{n}^{\circ}$ 93, 1990, p. 27-39.

Famille éducatrice, $\mathrm{n}^{\circ} 10$, décembre 1966, p. 3. 
toutes nos classes les accueillirent ${ }^{57}$. L'accueil des filles permet d'accroître les effectifs et par conséquent de mieux rentabiliser les locaux et d'amortir davantage le salaire des professeurs. L'application de la loi Debré renforce la pression sur les effectifs, même si dorénavant les enseignants sont rémunérés par l'État ${ }^{58}$.

\section{Les conséquences de la loi Debré}

Dans l'enseignement primaire, on l'a vu, la mixité a parfois été établie pour pallier le manque d'effectifs : dans un contexte d'exode rural, on regroupe filles et garçons afin de maintenir une école. Les contraintes budgétaires vont s'accroître du fait des contrats avec l'État permis par la loi Debré, votée en 1959. Certes, celle-ci représente un vrai soulagement financier, dans la mesure où les enseignants sont désormais rémunérés par l'État. Cependant, pour obtenir un contrat avec l'État, l'établissement privé doit avoir les effectifs suffisants. Cette contrainte est particulièrement nette dans l'enseignement secondaire, où s'ajoutent les effets de la réforme Fouchet ${ }^{59}$ : dans la plupart des cas, en effet, un seul établissement ne peut ouvrir les sept sections de terminale, elles-mêmes subdivisées en une multitude d'options; pour avoir les effectifs suffisants, l'établissement doit introduire la mixité. Pour nombre d'établissements, c'est une question de survie. Les parents d'élèves aux opinions les plus traditionnelles se trouvent pris, de ce fait, entre deux exigences contradictoires : d'un côté, ils souhaiteraient maintenir la séparation des sexes; d'un autre côté, ils tiennent à ce que l'établissement secondaire local présente le plus grand choix d'options possible : mieux vaut offrir toutes les filières (et donc accepter la mixité) que de mettre leurs enfants dans un établissement public.

Aussi bien la querelle entre partisans et adversaires de la mixité est-elle parfois considérée comme illusoire par les réalistes. Conscients des nécessités pratiques et financières, ceux-ci ne font pas l'apologie du principe de la mixité, mais la jugent inévitable. Ils dénoncent en outre le jusqu'au-boutisme de leurs adversaires, qui seraient prêts à fermer les écoles privées si elles devenaient mixtes : cela ne reviendrait-il pas à placer les enfants dans des écoles publiques

57 Ibid.

58 Sur la loi Debré, cf. Bruno Poucet (dir.), La Loi Debré. Paradoxes de l'Étatéducateur?, Amiens, CRDP de l'académie d'Amiens, 2001.

59 En 1965, les filières conduisant au baccalauréat sont spécialisées. Sont définis 4 baccalauréats généraux, les séries A, B, C, D (auquel s'ajoute le baccalauréat T, futur bac E), ainsi que trois baccalauréats de techniciens, les séries F, G, H (André D. Robert, L'École en France de 1945 à nos jours, Grenoble, Presses universitaires de Grenoble, 2010, p. 59). 
qui elles-mêmes seraient certainement mixtes? Autre faux dilemme, selon les réalistes : sous prétexte de refus de la mixité, imposée par des contraintes d'effectifs, doit-on répudier la loi Debré et ses salutaires avantages financiers? Mais comment vivre sans? La question de la mixité permet aux adversaires de la loi Debré de suggérer a posteriori qu'ils avaient eu raison de mettre en garde contre les risques de voir l'enseignement catholique perdre son âme ${ }^{60}$. Un éducateur conservateur comme Claude Courtois écrit ainsi :

"Sous la menace de voir s'écrouler l'enseignement libre, faute de moyens financiers, les contrats furent imposés à une majorité de parents, abusés par ce même argument ou tenus dans la plus complète ignorance.

Sous la menace de voir disparaître les contrats, condition de la survie d'un enseignement qui ne se dit même plus libre, la coéducation serait donc imposée pratiquement à ces mêmes parents?" ${ }^{61}$.

Cependant, Claude Courtois n'ose pas proposer franchement de rompre avec la logique de la loi Debré. Il se borne à préconiser de ne pas respecter le cadre de la réforme Fouchet (c'est-à-dire l'organisation des nouvelles filières du second cycle du second degré). Il admet également, conformément aux instructions de la Sacrée congrégation en date du 8 décembre 1957, qu'en cas de nécessité la mixité puisse être considérée comme un moindre mal. Mais ces cas doivent rester exceptionnels.

Mal acceptée par les milieux catholiques les plus intransigeants, la loi Debré, inversement, a scandalisé les milieux laïques, qui y ont vu une remise en cause de l'absence de subvention aux religions de la part de l'État ${ }^{62}$. Aussi bien la loi Debré a-t-elle rallumé la guerre scolaire, ravivant, dans certaines régions, la concurrence entre écoles publiques et écoles privées. Résultat : ce

60 Au moment des négociations sur le projet de loi sur l'enseignement privé, le secrétaire général Michel Descamps aurait voulu que les subventions de l'État fussent versées à une organisation nationale d'un enseignement privé qui serait resté indépendant; conformément à la logique de contre-société développée par les milieux cléricaux depuis les "lois laïques", les adversaires du projet dénoncent la menace de l'étatisme et préféreraient la résistance, quitte à ce que l'enseignement catholique perde des effectifs : la tentation du petit nombre... (Bruno Poucet, La Liberté sous contrat : une histoire de l'enseignement privé, Paris, Fabert, 2009, p. 77).

61 Claude Courtois, Coéducation et mixité, Paris, Le nœud de Carrick et l'Action scolaire, 1967, p. 13.

62 Seul fait partie du contrat, et donc est subventionné par l'État, ce qui relève de l'enseignement donné dans le respect des programmes scolaires définis par le ministère; catéchèse et pastorale sont à part et ne peuvent d'ailleurs, normalement, être imposées aux élèves. Le plus souvent, toutefois, les milieux laïques considèrent ces distinctions comme jésuitiques : les bâtiments ne sont-ils pas les mêmes, l'électricité payée dans un cadre ne sert-elle pas dans l'autre? Et, plus profondément, la conscience des enfants est-elle vraiment respectée dans le cadre de l'enseignement dispensé? 
sont parfois des représentants de l'enseignement public au sein des conseils départementaux de l'enseignement primaire qui se montrent réticents face à la mise en place de la mixité dans les écoles privées. En effet, selon l'article 36 de la loi du 30 octobre 1886, "aucune école privée ne peut, sans l'autorisation du conseil départemental, recevoir d'enfants des deux sexes, s'il existe, au même lieu, une école publique ou privée spéciale aux filles". Le 27 avril 1973, l'inspecteur d'académie des Bouches-du-Rhône est ainsi amené à écrire au ministère pour savoir ce qu'il faut entendre par le terme de "lieu». Le 9 juin, le ministère lui répond en spécifiant qu'il convient "de considérer la question dans le sens le moins restrictif possible», car "il est souhaitable que la mixité, déjà largement pratiquée dans l'enseignement public, se généralise "63. Indice de l'opposition des membres de l'enseignement public, la même question est posée par le préfet des Pyrénées-Atlantiques le 16 mai $1975^{64}$.

Se pose en outre le problème des pensionnats annexés. Selon le décret du 18 janvier 1887, il est de règle absolue qu'aucun pensionnat ne peut être annexé à une école primaire privée qui reçoit des enfants des deux sexes. Que faire en cas de gémination postérieure à l'annexion d'un internat? Le 17 juillet 1975 , l'inspecteur d'académie des Pyrénées-Atlantiques pose la question, au sujet des cours complémentaires de Salies-de-Béarn. Le 27 août, le ministère lui répond que l'internat de jeunes filles peut continuer à fonctionner en dépit de l'instauration de la mixité. Le ministère argumente en faisant remarquer que ce cours complémentaire "a reçu des élèves des deux sexes par suite de la généralisation de la mixité dans l'enseignement public et privé "65. Ainsi, le ministère favorise-t-il dans l'enseignement privé cette mixité qu'il va rendre obligatoire dans l'enseignement public l'année suivante (décrets des 26 et 28 décembre 1976).

\section{3 - L'aggiornamento de l'Église catholique}

L'évolution de l'enseignement catholique sur la mixité doit aussi être mise en relation avec l'aggiornamento de l'Église catholique à l'occasion du concile de Vatican II. Le 31 décembre 1929, par l'encyclique Divini illius Magistri, Pie XI avait proscrit fermement la coéducation, qualifiée d'erreur "pernicieuse". Au

63 AN, 19930639/1.

64 Ibid.

65 Ibid. 
demeurant, le texte pontifical condamnait également l'égalité d'éducation entre filles et garçons : "Il n'y a d'ailleurs dans la nature elle-même, qui a fait les sexes différents par leur organisme, par leurs inclinations, par leurs aptitudes, aucune raison qui montre que la promiscuité, et encore moins une égalité de formation, puissent ou doivent exister "66. Le principe de la séparation des sexes était jugé nécessaire à toutes les écoles, mais "principalement durant l'adolescence". Le 24 mars 1934, conformément à cette doctrine, était publiée une Déclaration des cardinaux et archevêques de France, à propos des "classes géminées " : "La morale enseigne que la juxtaposition prolongée des enfants de l'un et de l'autre sexe, à l'heure où les passions s'éveillent, constitue par elle-même un danger dont on doit avoir le souci ${ }^{67}$. Le 8 décembre 1957, les Instructions de la Sacrée congrégation des religieux sont une nouvelle condamnation de la coéducation : "La coéducation proprement dite ne peut d'une façon générale être approuvée en soi "68. Une évolution se dessine pourtant, puisque le texte admet que la coéducation peut présenter des avantages : elle peut continuer une honnête vie familiale, dans laquelle les jeunes gens de l'un et de l'autre sexe ne sont pas séparés, mais ont des relations décentes; elle peut favoriser l'émulation. Mais en définitive, la coéducation est jugée trop dangereuse pour les mœurs, si bien que les Instructions recommandent de respecter l'encyclique Divini illius Magistri. À défaut des moyens financiers pour maintenir la séparation des sexes, du moins faut-il respecter les prescriptions qui éviteront la promiscuité.

Le concile de Vatican II procède à un aggiornamento doctrinal et liturgique dont le retentissement est énorme. L'heure semble à l'ouverture au monde et à la modernité. Ce mouvement suscite la désapprobation des traditionalistes, en même temps que, chez des chrétiens progressistes, de nombreux espoirs. Ainsi des religieuses comme sœur Jeanne d'Arc et sœur Françoise Vandermeersch vont-elles s'appuyer sur le concile pour tenter de faire avancer leur point de vue féministe. "On peut dire, écrit Anthony Favier, que Vatican II a posé les fondements théologiques d'un féminisme chrétien catholique assumé voire revendiqué "69. Soucieuse d'une meilleure formation théologique des religieuses, sœur

66 En ligne:<http://www.vatican.va/holy_father/pius_xi/encyclicals/documents/hf_p-xi_enc_31121929 _divini-illius-magistri_fr.html>.

67 La Documentation catholique, n 97, 31 mars 1934.

68 La Documentation catholique, 30 mars 1958 (publication de documents issus des Acta Apostolica Sedis des 24-27 février 1958).

69 Anthony Favier, «Des religieuses féministes dans les années 68?», Clio. Histoire, femmes et sociétés, $\mathrm{n}^{\circ} 29,2009$, p. 69 . 
Jeanne d'Arc prône ainsi la mixité des études dans les universités catholiques. Concernant la mixité scolaire, le concile peut sembler moderniser la doctrine, au moins par omission, puisque la Déclaration conciliaire sur l'éducation du 28 octobre 1965, tout en faisant rituellement référence aux encycliques précédentes, ne dit rien sur la coéducation. Cette absence peut être interprétée comme le signe d'une évolution, conformément à l'adage "qui ne dit mot consent $»^{70}$. Les partisans de la mixité estiment que dans une déclaration conciliaire la référence aux textes antérieurs est une coutume (l'Église doit donner l'impression de la continuité de la doctrine), mais que cette référence préliminaire n'engage plus rien; qu'au demeurant l'encyclique de Pie XI fait allusion aux "différentes circonstances" et que les temps ont changé.

Cependant, l'absence de remise en cause explicite de l'ancienne doctrine peut favoriser les débats. Pour un adversaire de la mixité comme Claude Courtois, la déclaration conciliaire du 28 octobre 1965 fait encore référence à l'encyclique de 1929 : "ceux qui le nient sont comme les jansénistes qui niaient que le pape condamnât leurs erreurs " ${ }^{71}$. À ses yeux, comme aux yeux des opposants à la mixité, les principes énoncés par Divini illius Magistri sont intangibles, précisément parce que ce qui n'a pas changé, c'est la nature humaine : comme l'écrivait déjà Pie XI, la coéducation est fondée sur "un naturalisme négateur du péché originel». Le nier, pour le très conservateur magazine Défense du foyer, c'est reprendre tout simplement la doctrine de la franc-maçonnerie et préparer un "retour au paganisme", qui sera synonyme de complète dépravation sexuelle ${ }^{72}$. Les thuriféraires de la mixité prétendent qu'elle permet d'éviter rêveries et fantasmes malsains : "plusieurs jeunes filles, relève l'enquête menée par la Famille éducatrice en 1966, nous ont dit que la mixité les avait délivrées de l'obsession des garçons. [...] Le phénomène inverse est aussi reconnu $»^{73}$. Mais pour Claude Courtois, pas plus qu'aucune école n'est jamais parvenue à fermer une prison, pas plus la coéducation ne fera cesser les "dérèglements moraux ${ }^{74}$; le nier reviendrait à nier le poids du péché originel.

Sur la question de la coéducation comme sur d'autres, l'interprétation du

70 Témoignage d'Edmond Vandermeersch, 10 mars 2011.

71 Claude Courtois, Coéducation et mixité, op. cit. Claude Courtois est responsable de la Maison des œuvres, qui s'occupe des patronages et des colonies de vacances.

72 Défense du foyer, $\mathrm{n}^{\circ} 84$, février 1967, p. 2.

73 Famille éducatrice, $\mathrm{n}^{\circ}$ 10, décembre 1966, p. 3.

74 Claude Courtois, Coéducation et mixité, op. cit., p. 16. 
concile est donc source de polémiques et de malentendus dans les milieux chrétiens, entre progressistes et conservateurs. Aux yeux des traditionalistes, sans parler de l'aggiornamento doctrinal, avec la réforme de la liturgie, la fin de la messe en latin, la disparition rapide des servants, on ne fait pas que renoncer aux aspects festifs et émotionnels de la religion, on jette le bébé avec l'eau du bain, on s'écarte d'une tradition pluriséculaire pour se rapprocher d'un monde qui est pourtant de moins en moins chrétien ${ }^{75}$. De ce point de vue, la mixité est aussi un argument utilisé par les conservateurs pour dénoncer la rupture avec toutes les saines traditions. Leurs propos sont évidemment de nature à effrayer les parents qui se posent des questions. Aussi le Secrétariat général de l'enseignement catholique (SGEC) va-t-il finir par intervenir.

\section{Le SGEC prend position en faveur de la coéducation}

Les organisations s'appuyant sur l'encyclique Divini illius Magistri, et manifestant une hostilité absolue à l'égard de la mixité, peuvent profiter du désarroi de nombreux parents constatant que la mixité s'installe souvent sous l'emprise des nécessités pratiques sans avoir été discutée, et alors que longtemps les autorités ecclésiastiques s'étaient montrées réticentes.

"Depuis vingt ans et davantage que j'essaie, raconte le père mariste Rey-Herme, professeur à l'ISP, [...] de montrer qu'il serait peut-être intéressant de faire de l'éducation mixte, j'ai reçu un certain nombre d'interdictions de parler, j'ai été l'objet d'un certain nombre de rapports aux autorités religieuses, toujours au nom de l'encyclique de Pie XI présentant l'éducation mixte comme incompatible avec une perspective chrétienne. Mais ce qu'il y a de remarquable, c'est qu'il a suffi d'un problème financier pour qu'immédiatement Pie XI et les théologiens soient relégués au second rang et qu'on se mette à faire de l'éducation mixte ${ }^{76}$.

De nombreux parents sont donc inquiets et se demandent s'ils font bien d'envoyer leurs enfants dans un établissement mixte. C'est pourquoi le Secrétariat général de l'enseignement catholique (SGEC) décide prendre position en publiant, le $1^{\text {er }}$ juin 1966, une note approuvée, et c'est important, par la Commission épiscopale du monde scolaire. Ce texte est ratifié par le Comité national de l'enseignement catholique (CNEC), véritable parlement de l'enseignement

75 Gérard Cholvy, Yves-Marie Hilaire, La France religieuse, reconstruction et crises, 1945-1975, Toulouse, Privat, 2002, p. 102-108.

Mixité et coéducation, Dossier CDM, n85, Centre de documentation mariste, mars 1969, p. 3. 
catholique $^{77}$, lors de sa session des 18 et 19 février $1967^{78}$. La note vise d'abord à lever un tabou et à rassurer les parents ${ }^{79}$. Mais le secrétaire général adjoint de l'enseignement catholique, Edmond Vandermeersch, ne veut pas s'en tenir là : il entend aussi encourager une réflexion sur une mixité qu'il ne faut pas seulement subir. À vrai dire, il s'est montré particulièrement volontariste, car la plupart des partenaires de l'enseignement catholique sont réticents ${ }^{80}$. Le président des APEL (asssociations des parents d'élèves de l'enseignement libre), M. Laune, qui se fait le porte-parole d'une conception traditionnelle de la famille, se montre très réservé. Il en va de même de $\mathrm{M}^{\mathrm{gr}}$ Descamps, ancien secrétaire général de l'enseignement catholique, devenu représentant de l'enseignement catholique français auprès de l'Office international de l'enseignement catholique, qui est persuadé que la cohabitation de jeunes gens des deux sexes dans les mêmes établissements relève de l'inconscience et qui n'imagine pas qu'il puisse exister une pédagogie de la coéducation. MM. Courau et Charmassin, qui représentent l'enseignement agricole au CNEC (notamment les Maisons familiales rurales), estiment que les milieux agricoles ne sont pas prêts et qu'ils restent marqués par la division sexuelle des tâches. Même le président de la FEP-CFDT, Charles Brunold, insiste sur la nécessité de la discrétion; il ne croit "pas indispensable de généraliser " les expériences en cours ${ }^{81}$. Le président du Syndicat national des chefs d'établissement d'enseignement libre (SNCEEL), M. Ancel, a une position attentiste. En dépit de ces réserves, Edmond Vandermeersch parvient à faire voter par le CNEC, au cours de cette session de février 1967, une résolution approuvant la note du SGEC du $1^{\text {er }}$ juin 1966 sur la mixité.

Cette note est prudente, mais favorable à l'évolution vers la mixité. Répondant aux critiques qui sont faites par les adversaires de la mixité, elle insiste sur le fait que cette dernière n'est pas seulement la conséquence de la loi Debré, mais du changement des modes de vie :

"Il faut d'ailleurs concevoir l'éducation donnée aux jeunes en fonction d'un monde où les loisirs, la vie culturelle et sociale, l'activité professionnelle ne connaissent

77 Sur les statuts et la composition du CNEC, cf. Edmond Vandermeersch, École : Église et laïcité. Souvenirs autour de la loi Debré (1960-1970), Paris, L'Harmattan, 2008, p. 61-66.

78 Archives départementales de la Somme [désormais AD Somme], Fonds Vandermeersch, 77J41.

79 Témoignage d'Edmond Vandermeersch, 10 mars 2011.

80 Ibid.

81 La prudence de la FEP-CFDT résulte peut-être de la nécessité de se montrer modérée, après sa rupture avec la CFTC. Sur la FEP-CFDT, cf. Bruno Poucet, Entre l'Église et la République. Une histoire de la Fédération de la formation et de l'enseignement privé CFDT, Paris, Éd. de l'Atelier, 1998. 
plus les barrières d'autrefois. Même si les circonstances ne l'imposaient pas, on pourrait donc envisager de s'orienter volontairement vers la mixité afin de proposer aux jeunes un milieu éducatif plus conforme aux conditions de la vie extra-scolaire d'aujourd'hui. Les méthodes d'éducation pourraient trouver là une source de renouveau $" 82$.

Assurément, le SGEC ne représente pas, pour les établissements privés, ce que le ministère de l'Éducation nationale représente pour les établissements publics : il n'a pas autorité sur eux, sa déclaration n'est qu'une recommandation. Il n'en reste pas moins que, fait nouveau, les responsables de l'enseignement catholique, avec l'appui des évêques ${ }^{83}$, se prononcent officiellement en faveur de la mixité. Ce terme de "mixité» est d'ailleurs retenu seulement parce qu'il est alors consacré par l'usage, mais c'est une véritable coéducation que le SGEC recommande de mettre en place dans sa note du $1^{\text {er }}$ juin 1966 :

"Là où pour des raisons purement administratives, on se contente de faire vivre ensemble garçons et filles sans mettre en œuvre ni une co-instruction ni une co-éducation, il en résulte pour les jeunes plus d'inconvénients que d'avantages. Responsables et éducateurs qui s'orientent vers la mixité des établissements ont le devoir grave de mettre en œuvre une véritable co-éducation qui réponde aux exigences pédagogiques précises de cette situation nouvelle " ${ }^{84}$.

L'insistance sur la coéducation est donc liée à des "exigences pédagogiques ", ce qui n'est pas étonnant dans la mesure où l'instigateur de cette résolution, Edmond Vandermeersch, s'intéresse beaucoup aux questions pédagogiques. De fait, la note du SGEC multiplie les recommandations. La première est que, en dehors des écoles primaires, le corps enseignant d'un établissement mixte soit lui-même mixte. Il en va de même pour sa direction. "S’il n’est pas nécessaire qu'une telle co-direction soit juridiquement établie, du moins faut-il qu'elle soit officiellement reconnue ${ }^{85}$. La seconde préconisation est d'éviter que les effectifs des élèves de l'un ou de l'autre sexe soient disproportionnés par rapport à l'autre. "La proportion du tiers semble le minimum indispensable, l'idéal étant que les effectifs de garçons et de filles se trouvent à peu près équivalents " ${ }^{86}$. Le SGEC

82 AD Somme, Fonds Vandermeersch, 77J41.

83 Les évêques, qui ne tenaient pas à exprimer une position publique, ont laissé faire (témoignage d'Edmond Vandermeersch, 10 mars 2011).

84 AD Somme, Fonds Vandermeersch, 77J41.

85 Note du SGEC du $1^{\text {er }}$ juin 1966, AD Somme, Fonds Vandermeersch, 77J41.

86 Ibid. 
recommande par ailleurs de ne jamais faire débuter la mixité en cours de cycle, en quatrième ou en troisième par exemple : "en dehors des classes élémentaires, elle peut commencer soit en sixième, soit en seconde. Elle sera plus difficile à réaliser en seconde lorsque le second cycle ne constitue pas un établissement indépendant des classes de premier cycle qui, elles, ne seraient pas mixtes ${ }^{87}$. Des cas existent, en effet, d'établissements qui accueillent des élèves des deux sexes seulement dans le second cycle du second degré, à la fois pour des raisons d'effectifs et pour pallier l'absence d'établissement privé de second cycle pour l'un des deux sexes. Face à ces raisons de commodités, dénuées de réflexion pédagogique, le SGEC insiste : "Lorsque la mixité est décidée, mieux vaut la généraliser dans tout un ensemble à la fois, au moins un cycle scolaire tout entier, que de la maintenir dans quelques classes seulement». Le SGEC recommande par ailleurs que dans un établissement mixte le cadre éducatif (récréations, etc.) ainsi que les activités non scolaires (vie liturgique, enseignement religieux) soient également mixtes. Dernière recommandation du SGEC : "les professeurs doivent se persuader que l'on ne fait pas la classe à un groupe mixte de la même façon qu'à des garçons ou à des filles ${ }^{88}$. De fait, cette prise en compte des caractères féminins et masculins et de leur rencontre fait l'objet de nombreuses réflexions, qui témoignent de la persistance de l'idée de natures différentes.

\section{La rencontre des caractères masculins et féminins}

Pour les catholiques, il existe des caractères masculins et féminins, deux natures conçues différemment lors de la création. Faire cours dans des classes mixtes implique d'en tenir compte.

\section{Enseigner en tenant compte de la différence des caractères masculin et féminin}

Il y a toujours un décalage entre la décision d'introduire la mixité et la mixité effective des classes. Au départ, les parents conservent leurs habitudes. En 1970, Antoinette Salmon-Legagneur décide d'introduire la mixité dans l'établissement qu'elle dirige à Paris rue de Rocroy. Mais elle a du mal, dans un premier temps, à recruter des jeunes gens car, si certains parents voient d'un

\footnotetext{
87 Ibid.

88 Ibid.
} 
bon œil l'envoi de leurs filles dans des établissements de garçons, l'inverse est souvent considéré comme un déclassement. Aussi bien, au début, les directeurs des établissements voisins ne lui envoient-ils que des élèves difficiles ${ }^{89}$. Au demeurant, les parents ne sont pas toujours empressés d'envoyer leurs filles dans un ancien établissement de garçons : ils craignent que leur fille ne soit mêlée à trop de garçons. Lorsque le lycée de La Providence d'Amiens s'ouvre à la mixité, en 1973-1974, "la préoccupation était plutôt de savoir où trouver des candidates à la mixité... qui ne redoutaient pas d'être très minoritaires", se souvient le père Bouzy ${ }^{90}$. Il n'y a donc que sept filles la première année; on les regroupe dans la même classe de sixième, afin qu'elles ne se sentent pas trop perdues parmi les garçons. Vingt ans plus tard, quand, en 1988, le second cycle du lycée Saint-François-de-Sales de Troyes s'ouvre aux garçons, la classe de seconde compte un garçon pour quatre-vingt-six filles. L'inscription des garçons est progressive dans les années qui suivent; en l'occurrence, les bons résultats au baccalauréat de l'établissement ont été un facteur attractif. Dans un cadre concurrentiel, non contraint par un zonage géographique, la réputation de l'établissement joue un rôle important.

À l'école-collège Notre-Dame de Reims, où la mixité a été introduite en 1979, les garçons sont au départ très minoritaires. L'équilibre entre les effectifs masculins et les effectifs féminins a été atteint plus rapidement dans la partie école que dans la partie collège (Voir la figure ${ }^{91}$ page suivante).

L'introduction de la mixité vaut pour la vie scolaire (pas de séparation dans la cour de récréation), la catéchèse et la vie pastorale, ainsi que pour les activités extra-scolaires. Signe de la radicalité du passage à la mixité, en 1975, au lycée de la Providence d'Amiens, dans le cadre de la politique des " $10 \%$ »" la direction, alors même que la mixité n'a été introduite que deux ans auparavant, a accepté que les élèves organisent des sorties à vélo avec leurs professeurs : garçons et filles partent dans une ferme de la région d'Abbeville, d'où ils rayonnent pour découvrir la région ${ }^{93}$.

89 Antoinette Salmon-Legagneur, témoignage du 20 janvier 2011.

90 Témoignage du père Dominique Bouzy, 22 avril 2014.

91 Graphique établi d'après les statistiques communiquées par Claude Pouphile, ancienne directrice de l'établissement, que je remercie pour son obligeance.

92 En 1973, le ministre Joseph Fontanet a décidé une réduction de $10 \%$ de tous les programmes, l'horaire libéré étant mis à la discrétion des établissements.

93 Témoignage de Sabine Perreau, 22 avril 2014. 


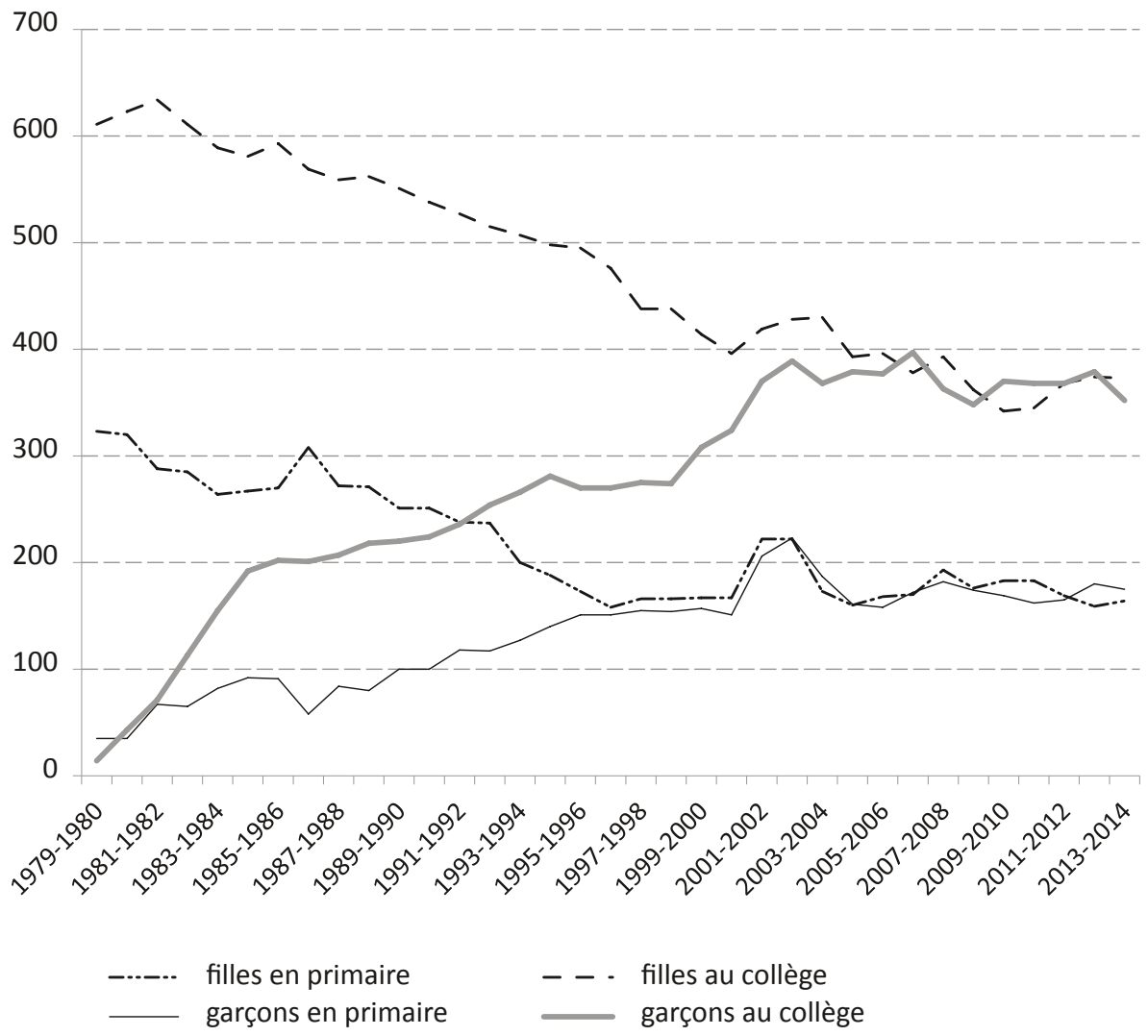

Évolution des effectifs de l'école primaire élémentaire et du collège Notre-Dame de Reims depuis l'introduction de la mixité, 1979-2014

Le passage à la mixité conduit par ailleurs à de petites modifications concrètes. À l'école-collège Notre-Dame de Reims, les petites filles portaient des blouses roses. Avec l'introduction de la mixité, en 1979, on passe à une couleur bleu jean, qui apparait moderne et unisexe. Les toilettes sont par ailleurs réaménagées ${ }^{94}$. Au lycée Saint-François de Troyes, qui organise des représentations théâtrales, ce sont désormais des garçons qui jouent les rôles masculins dans les pièces de théâtre, ce qui satisfait les filles pour la bonne raison qu'elles répugnaient auparavant à endosser les rôles masculins ${ }^{95}$. Au lycée de la Providence, à Amiens, ancien établissement de garçons, les pro-

94 Témoignage de l'ancienne directrice, Claude Pouphile, 31 mars 2014.

95 Témoignage de sœur Geneviève Poinsot, 12 mars 2014. 
fesseurs qui avaient l'habitude d'appeler les élèves par leur nom, se mettent progressivement à utiliser les prénoms ${ }^{96}$.

Ce changement d'habitude va dans le sens de l'idée couramment admise que l'introduction des filles dans un établissement de garçons contribue à donner moins d'âpreté aux relations entre les individus. L'idée que la mixité permettrait de réduire les défauts masculins et féminins, car on trouverait "plus de simplicité chez les unes, moins de rudesse et de grossièreté chez les autres ${ }^{97}$, et donc qu'elle améliorerait l'ambiance de travail, est un poncif d'articles de journaux ou de revues. Est-elle corroborée par les témoignages? Selon Claude Pouphile, ancienne directrice de l'école-collège Notre-Dame à Reims, le passage à la mixité a bien joué en ce sens. Elle estime qu'avec la mixité, les filles se comportent moins entre elles comme des "chipies "98. Pour sa part, sœur Geneviève Poinsot, ancienne directrice du lycée Saint-François de Troyes, souligne qu'il ne faut pas idéaliser et que l'arrivée des garçons dans un ancien établissement pour jeunes filles a parfois pu donner au personnel l'impression que l'atmosphère, dans les couloirs ou dans la cour de récréation, était devenue moins policée; le personnel a dû apprendre à parler durement quand il le fallait ${ }^{99}$. La différence d'appréciation entre les deux témoignages provient-elle de ce que la mixité a été établie dans un cas en 1979, et dix ans plus tard dans l'autre?

Il est des cas où la mixité des élèves a été précédée de la mixité du corps enseignant. Il en a été ainsi à l'école-collège Notre-Dame de Reims, où, dans un esprit d'ouverture sur la vie, la directrice Claude Pouphile avait recruté quelques hommes dans le corps enseignant ${ }^{100}$. Par ailleurs, dans un établissement passé tardivement à la mixité comme Saint-Joseph de Reims, la mixité du personnel a été antérieure à celle des élèves (1993), car l'établissement ne pouvait refuser le mouvement général de féminisation du personnel sans pratiquer de discrimination à l'embauche ${ }^{101}$. Dans beaucoup d'établissements cependant, l'absence de mixité des élèves est longtemps allée de pair avec celle des enseignants, la première n'entraînant pas mécaniquement la seconde car il a fallu attendre que des postes se libèrent. Au lycée de La Providence d'Amiens, le personnel est uniquement

96 Témoignage de Sabine Perreau, 22 avril 2014.

97 Famille éducatrice, art. cit., p. 3.

98 Témoignage de Claude Pouphile, 31 mars 2014.

99 Témoignage de sœur Geneviève Poinsot, 12 mars 2014.

100 Témoignage de Claude Pouphile, 31 mars 2014.

101 Témoignage d'Odile Pollet du 2 avril 2014. 
masculin l'année où est introduite la mixité (1973-1974). Les femmes sont deux religieuses, l'une à la porterie, l'autre à l'infirmerie. "Nous n'hésitions pas à aller discuter avec elles", se souvient Sabine Perreau, qui appartint à la première promotion mixte et qui ajoute par ailleurs que "les jésuites étaient attentifs et ouverts " ${ }^{102}$. Au collège-lycée Saint-Martin de Rennes, tenu par les pères eudistes, le personnel reste d'abord largement masculin ${ }^{103}$. Dans ces deux cas, la féminisation du personnel est surtout, par la suite, un effet de la sécularisation du personnel et du plus grand recrutement de personnel enseignant féminin, phénomène qui vaut pour le privé comme pour le public. En général, le passage à la mixité a néanmoins conduit au recrutement immédiat, dans les anciens établissements pour garçons, de professeurs femmes pour enseigner la gymnastique aux jeunes filles, et, à l'inverse, de professeurs hommes dans les anciens établissements pour jeunes filles ${ }^{104}$. Par ailleurs, pour certains anciens établissements féminins, le recrutement de professeurs hommes a pour but d'attirer la clientèle. Ainsi, au collège Saint-Vincent-de-Paul (Paris $10^{\mathrm{e}}$ ), Antoinette Salmon-Gagneur prend soin, au moment où elle décide d'ouvrir l'établissement aux garçons, de recruter des professeurs masculins, plus prestigieux aux yeux des parents ${ }^{105}$. La réflexion relève ici des représentations en terme de niveau scolaire.

Il y a pu y avoir, toutefois, une véritable réflexion pédagogique précédant le passage à la mixité. C'est surtout vrai pour les passages opérés après 1970 . Avant cette date, le souci des effectifs a souvent été le motif du passage à la mixité, et c'est d'ailleurs pourquoi le SGEC a poussé à ce que soit mise en œuvre dans les établissements une réflexion sur la coéducation. À l'école-collège Notre-Dame de Reims, le personnel a réfléchi pendant deux ans aux changements qu'il allait falloir opérer une fois la mixité établie. Du point de vue pédagogique, c'est souvent l'enseignement du français qui revient dans les écrits de l'époque, comme dans les témoignages rétrospectifs, avec l'idée que garçons et filles n'ont pas les mêmes centres d'intérêt. Les témoignages que nous avons pu recueillir signalent souvent que les professeurs de français ont eu l'impression d'avoir des classes plus vivantes, car enrichies de points de vue

102 Témoignage du 22 avril 2014. Sabine Perreau est actuellement présidente de l'association des anciens élèves.

103 Témoignage de Quitrie Benvenuti, 19 mars 2014.

104 Sabine Perreau signale cependant qu'à Amiens, la première année (1973-1974), les cours d'EPS étaient mixtes, aussi bien pour la gymnastique que pour le football; par la suite, a été recrutée une enseignante particulière pour les filles (témoignage du 22 mars 2014).

105 Antoinette Salmon-Legagneur, témoignage du 20 janvier 2011. 
différents. En 1966, dans Famille éducatrice, le directeur du collège mixte de l'Assomption de Briey, M. Millard, qui est aussi professeur de français, rapporte qu'il demande aux garçons s'ils admettent que l'Alceste du Misanthrope puisse haïr l'humanité et aimer pourtant Célimène, et qu'une classe mixte enrichit ce genre de débats, car mieux vaut connaître les Célimène que de les imaginer ${ }^{106}$. $\mathrm{Au}$ demeurant, des professeurs enseignant dans des classes non mixtes ont eux-mêmes considéré que la mixité de la vie sociale devait les amener à modifier leurs pratiques. Interrogée par le mensuel Pédagogie. Éducation et culture, une religieuse enseignant le français et la philosophie explique ainsi qu'elle a modifié ses cours. Auparavant, elle présentait de façon générale les questions de l'éducation du cœur et des sens.

"J'ai été amenée bien plus tôt qu'autrefois à parler aux grandes comme à des femmes qui ont déjà découvert l'homme. En français, j'ai insisté davantage sur la psychologie des poètes, leurs déceptions amoureuses, leurs conceptions de l'amour et de la femme. En philosophie, j'ai montré la différence entre la psychologie masculine et la psychologie féminine. Les problèmes du couple s'articulent sur ceux de la communication des consciences. L'étude de l'amour a, je le sens, une résonance beaucoup plus concrète qu'autrefois "107.

Pour certains enseignants, toutefois, la mixité est mauvaise d'un point de vue pédagogique, car on n'éduque pas une fille comme un garçon. "Ils sont trop totalement différents, estime une autre religieuse interrogée par le mensuel Pédagogie. Éducation et culture. Il y a des réflexions que l'on peut faire devant un auditoire masculin, non devant de petites filles. La manière même est différente. Il faut être tellement plus souple, plus "fin" - au sens pascalien du mot - avec les filles. La tâche de l'éducatrice est déjà assez compliquée comme ça, sans y ajouter cette difficulté supplémentaire d'adaptation à une matière première si différente „108. La réticence à enseigner dans un établissement mixte peut donc provenir, non pas de la crainte des dangers moraux, mais de l'impression qu'il sera plus difficile d'enseigner, avec l'idée que garçons et filles n'envisagent pas les problèmes sous le même angle, car leur maturité est différente. Une autre écrit d'ailleurs dans la même revue : "Si on arrive à façonner un public homogène, c'est en niant la différence des sexes, et ceci

106 Famille éducatrice, $\mathrm{n}^{\circ}$ 10, décembre 1966, p.3.

107 Pédagogie. Éducation et culture, novembre 1965, p. 878.

108 Ibid., p. 873. 
ne me semble pas tellement souhaitable ${ }^{109}$. En l'occurrence, ce point de vue est aussi une arme contre la mixité, même s'il importe effectivement pour les catholiques de veiller au respect de la différence de nature entre l'homme et la femme. Car si la déclaration conciliaire Gravissimum educationis momentum du 28 octobre 1965 n'insiste pas sur la nécessité de séparer les sexes, elle prescrit en revanche de tenir compte, dans l'éducation, de la différence des sexes et de la "vocation particulière attribuée à l'homme et à la femme, par la divine Providence, dans la famille et la société "110.

\section{2 - Le respect des "natures" masculine et féminine}

La question de ce qui est considéré comme un risque de dénaturation est omniprésente dans le discours des éducateurs catholiques sur la mixité. Dans l'enquête menée en 1965 par le mensuel Pédagogie. Éducation et culture (donc avant la note du SGEC de 1966), un chef d'établissement mixte avoue ainsi qu'existe "le risque de "viriliser" à l'excès les jeunes filles qui sacrifient un peu de leur grâce, de leur sens esthétique, à d'autres qualités estimables, et mêmes utiles, mais moins spécifiques à leur sexe ${ }^{111}$. Les contempteurs de la mixité insistent évidemment sur ce risque, tel Défense du foyer, pour qui "la mixité produit des "camarades”, c'est-à-dire des "garçons-filles" et des "filles-garçons” "112. Pour sa part, Claude Courtois suggère que les garçons risquent de devenir homosexuels, en développant leurs caractères "féminoïdes" $(s i c)^{113}$. Mais les partisans de la mixité évoquent aussi la question, ne serait-ce que pour minimiser les risques. Ainsi Marc Harteman observe que dans les établissements mixtes, filles et garçons forment des groupes séparés, "qui ont leur conscience propre, générique (sic), si l'on ose dire; leur féminité, leur masculinité, soigneusement entretenue, jalousement cultivée parfois de part et d'autre "114. Cette distinction entre mixité et mélange peut sembler une étrange conception de la mixité. Pour leur part, plutôt que de coéducation, les Guides de France préfèrent d'ailleurs se référer à l'«interéducation", c'est-à-dire à l'éducation à la différence sexuée et à la

109 Ibid., p. 880.

110 Cité par Marcel Launay, L'Église et l'École en France, XIX ${ }^{e}-X X^{e}$ siècles, Paris, Desclée, 1988, p. 19.

111 Pédagogie. Éducation et culture, novembre 1965, p. 893.

112 Défense du foyer, février 1967, p. 8.

113 Claude Courtois, Coéducation et mixité, op. cit., p. 16-17. On observera qu'alors même qu'il insiste sur le caractère intangible de la nature humaine, pécheresse, Claude Courtois croit néanmoins possible la dénaturation, dès lors qu'il s'agit de différence entre les sexes.

114 Marc Harteman, art. cit., p. 17. 
coopération harmonieuse entre les sexes. Dans les établissements d'enseignement qui ont été conduits à adopter la mixité, il est vraisemblable qu'il en soit souvent de même. Certes, les directives du SGEC appellent à la coéducation, mais c'est plutôt l'interéducation qui est l'orientation générale.

Même si les témoignages montrent que les professeurs des classes non mixtes ont bien plus peur d'un amoindrissement des caractères sexués que leurs collègues des classes mixtes, il n'en reste pas moins que tous les éducateurs catholiques considèrent qu'il existe une nature propre à chaque sexe. Cette conception est conforme à la doctrine réaffirmée par Vatican II, qui rappelle que les maîtres doivent savoir "tenir compte, dans toute l'éducation, de la différence des sexes et du but attribué à chacun par la Providence divine, dans la famille et la société». Face aux contempteurs de la mixité rappelant que Pie XI avait dénoncé dans la coéducation la marque du "nivellement égalitaire", ses partisans insistent sur l'idée que la mixité ne repose pas sur la négation de la nature propre à chaque sexe. "Dans la mixité telle qu'elle doit être envisagée, écrit Antoine de La Garanderie dans une mise au point publiée par Famille éducatrice, il est bien entendu qu'elle ne se fonde pas sur le naturalisme égalitaire ni sur la négation du péché originel ${ }^{115}$. Une déclaration du Comité national de l'enseignement catholique dénonce de même, lors de la session des 18 et 19 février 1967, la confusion souvent faite entre le "naturalisme pédagogique" (qu'il définit comme "la non distinction des vocations propres à chaque sexe et la négation du péché originel et de l'action de la grâce") et la "coéducation qui se fait pour les garçons et les filles réunis dans un même établissement "116. Le SGEC recommande d'ailleurs de veiller à conserver des temps éducatifs au cours desquels les sexes sont séparés.

Cette insistance sur l'idée de nature spécifique à chaque sexe n'est pas propre aux éducateurs qui s'intéressent au milieu scolaire. Ainsi, lorsqu'en 1966, Guides de France et Scouts de France entreprennent de créer les "Jeunes en marche", ils précisent qu'ils sont "conscients de la spécificité profonde des filles et des garçons ${ }^{117}$. L'UFCS, qui met l'accent sur la différence et la complémentarité, insiste également sur la vocation propre à chaque sexe. Ce discours n'est certes

115 Famille éducatrice, nº 3 , mars 1967, p. 3.

116 AD Somme, Fonds Vandermeersch, 77J41.

117 Extrait de l'éditorial commun à la revue La Route et à la revue Feux nouveaux de juillet 1965, cosigné par Odile Bonte et Jean Vigier (cité par Marie-Thérèse Cheroutre, Le Scoutisme au féminin : les Guides de France, 1923-1998, op. cit., p. 364). 
pas une spécificité des milieux catholiques ${ }^{118}$. Il contribue néanmoins à expliquer qu'il ne soit pas question, dans la réflexion menée par les milieux catholiques sur la mixité dans l'enseignement, de lutter contre les stéréotypes sexuels (qui sont d'ailleurs régulièrement repris lors des débats, même par ceux qui se montrent partisans de la mixité). Mathilde Dubesset estime que cette insistance sur la complémentarité homme/femme a conduit les catholiques à parler, davantage que les protestants, de la différence, et moins de l'égalité des sexes ${ }^{119}$. Pour la revue de l'UFCS, La Femme dans la vie sociale, il y a des "métiers féminins" permettant aux femmes de développer leurs "dons naturels", car "la nécessaire formation professionnelle ne doit pas empêcher d'être femme avant tout „ ${ }^{120}$. Pour le SGEC, il n'est donc pas question de combattre les inégalités d'orientation scolaire entre les sexes. Il importe au contraire de respecter la "vocation" de chaque sexe. C'est ce que déclare sa note sur la mixité du $1^{\text {er }}$ juin 1966 :

"Programmes et méthodes d'enseignement dans un établissement mixte doivent tenir compte des orientations scolaires et professionnelles qui habituellement distinguent les garçons et les filles. Les uns s'orientent volontiers vers les sections scientifiques et les classes techniques industrielles; les autres vers les sections littéraires et celles de techniques administratives ou sociales. Une classe mixte dès le premier cycle ne peut donc privilégier le travail des sciences ou celui des lettres, elle doit favoriser également l'un et l'autre afin d'assurer à chacun des groupes les meilleures chances de réaliser sa vocation propre "121.

Pour Antoine de La Garanderie, la mixité implique assurément l'égalité, si celle-ci est conçue comme une "égalité de la dignité personnelle». Mais cela n’a rien à voir avec le "naturalisme égalitaire", car les éducateurs exerçant dans des classes mixtes doivent conserver l'idée que "l'homme et la femme ont des vocations propres. La mixité n'implique pas qu'on l'oublie»"22. L'égalité n'est pas l'identité. Au demeurant, comme l'explique le $\mathrm{D}^{\mathrm{r}}$ Le Moal dans sa conférence du 15 décembre 1967 à l'Institut catholique, "le fait que les deux sexes puissent accomplir les mêmes tâches ne prouve pas qu'il y ait identité des sexes, car l'un et l'autre peuvent arriver au même résultat avec des moyens et des aptitudes différentes, en s'y prenant différemment. C'est pourquoi poser le problème sous

118 Sylvie Chaperon, Les Années Beauvoir, 1945-1970, op. cit., Paris, Fayard, 2000, p. 338-339.

119 Mathilde Dubesset, art. cit., p. 29-30.

120 La Femme dans la vie sociale. Notre journal, novembre 1956, cité par Mathilde Dubesset, art. cit., p. 29.

121 AD Somme, Fonds Vandermeersch, 77J41.

122 Famille éducatrice, nº 3 , mars 1967, p.3. 
l'angle supériorité-infériorité parait sans fondement "123. Ainsi la question de l'égalité est-elle éludée pour mieux insister sur la différence d'identité. Dans Panorama chrétien de septembre 1967, Jeanne-Marie Fauré va dans le même sens :

"Quant à la non-différenciation des sexes, voilà une idée qui fera bientôt sourire. Hommes et femmes nous sommes, et le nivellement, voire l'égalité, sont des termes de suffragette. Une femme vaut bien un homme, c'est sûr, mais elle lui est si différente que dans bien des cas... elle vaut plus " ${ }^{124}$.

Rétrospectivement, l'ancien secrétaire général de l'enseignement catholique, Edmond Vandermeersch, admet que "le problème de l'inégalité entre les hommes et les femmes n'était pas du tout dans les débats". Il reconnait que l'affirmation théologique de l'égalité de dignité a pu occulter les inégalités de fait : "On pensait qu'il fallait encourager les filles vers leurs voies naturelles". Mais il estime que la question de l'égalité entre les sexes et de la lutte contre les stéréotypes au moment de l'orientation, n'était pas, à cette époque, davantage mise en avant dans l'enseignement public. Lui-même croisait souvent des inspecteurs primaires et des inspecteurs pédagogiques régionaux ${ }^{125}$ qui étaient plus "moralistes" que lui sur la question de la mixité ${ }^{126}$.

Est-ce à dire qu'il n'y aurait finalement aucune différence entre le secteur public et le secteur privé, que les mêmes préjugés et les mêmes attitudes existeraient dans les deux cas? Il existe de fait des maîtres de l'enseignement public qui se satisfont très bien de la séparation des sexes. À Coutances, l'école primaire du centre ville (l'école "de la rue Saint-Maur"), qui accueille plutôt des enfants des classes moyennes, ne devient mixte qu'en 1977, du fait de l'obligation légale, et ce n'est pas seulement un effet de la concurrence des écoles privées catholiques et de la crainte d'effaroucher les parents de la cité épiscopale. Le corps enseignant lui-même se soumet à contrecœur à l'obligation de la mixité. Par ailleurs, d'après les témoignages que nous avons pu recueillir, les représentations des inspecteurs primaires (qui deviennent en 1969 les

123 Centre catholique d'éducation familiale ACGF et ACGH, Mixité et coéducation, op. cit., p. 14.

124 Panorama chrétien, art. cit., p. 68.

125 Inspecteurs pédagogiques régionaux. Le corps des IPR a été créé en 1964 pour suppléer les inspecteurs généraux, qui n'étaient plus assez nombreux pour inspecter les professeurs de l'enseignement secondaire, pour deux raisons : d'une part du fait de la démocratisation/massification du second degré, d'autre part du fait de la loi Debré de 1959, car les enseignants des établissements sous contrat sont inspectés par les inspecteurs de l'État, dans les mêmes conditions que leurs collègues des établissements publics.

126 Témoignage du 10 mars 2011. 
inspecteurs départementaux de l'Éducation nationale-IDEN) sur l'orientation scolaire des garçons et des filles reflétaient souvent les stéréotypes de genre. Il faudrait assurément savoir quelle est la valeur de ces témoignages. Il semble y avoir tout de même une différence avec l'enseignement catholique : n'est pas exprimé le souci de préserver la nature féminine. Les maîtres du public qui redoutent l'introduction de la mixité craignent la distraction des élèves, un moindre sérieux dans les apprentissages scolaires. La question est celle de l'efficacité scolaire. Il en va de même des conceptions des IDEN sur l'orientation : s'ils reconnaissent maintenant que leurs conceptions étaient empreintes de préjugés, ils considèrent néanmoins qu'ils agissaient en fonction de ce qu'ils estimaient être la voie la plus efficace et non dans l'optique de préserver une quelconque nature, a fortiori une nature issue de la création.

L'enseignement privé s'oriente vers la mixité un peu plus tardivement que l'enseignement public. Perdure longtemps la tradition de "protéger" les jeunes filles. Néanmoins, les années 1960 et 1970 représentent un tournant. En 1970, près de la moitié des établissements primaires privés sont mixtes. Pour l'enseignement secondaire, il n'est pas possible, faute de chiffres officiels, de prendre la mesure statistique de la mutation, mais elle est évidente. Dans le cas de cet enseignement, il semble toutefois qu'il faille distinguer deux phases : au cours de la première, celle des années 1960, ce sont les considérations d'effectifs, en particulier après la loi Debré, qui ont conduit à l'établissement de la mixité; au cours de la seconde, celle des années 1970, il s'agit davantage d'un souci d'ouverture au monde tel qu'il est. Cependant, grande est la diversité des situations, selon le contexte géographique et social propre à chaque établissement, la concurrence avec d'autres établissements, voire les traditions des congrégations.

Pendant la première phase, les établissements semblent agir sous la contrainte d'impératifs financiers. Aussi le passage à la mixité suscite-t-il de vifs débats. Les uns s'y résignent, tout en pensant que la coéducation ne va pas sans risque de perturbations psychologiques et intellectuelles, à l'âge critique de l'enfance et de l'adolescence : mieux vaudrait que garçons et filles se forment et se développent séparément. D’autres applaudissent et considèrent, au contraire, que dans un monde où ils sont amenés à se côtoyer de plus en plus, les enfants des deux sexes ne peuvent que gagner à vivre entre eux à l'école. Les plus traditionalistes, enfin, continuent de se référer à l'encyclique Divini illius magistri. En fait, il existe un décalage entre les intellectuels progressistes favorables à la mixité et les 
milieux catholiques traditionnels : lors du débat qui a lieu au Comité national de l'enseignement catholique, la majorité des organisations représentatives de l'enseignement catholique se montrent réticentes. Et pourtant, sous l'impulsion de secrétaire général adjoint de l'enseignement catholique, Edmond Vandermeersch, proche des pédagogues progressistes, le SGEC décide de prendre position en faveur de la mixité, souhaitant qu'il soit fait de nécessité vertu et que la mise en place de la mixité soit l'occasion d'une réflexion pédagogique sur la coéducation. À la différence des adversaires de la mixité, qui insistent sur le poids du péché originel, le SGEC va donc dans le sens de ceux qui se montrent convaincus d'une éducabilité entre les sexes. Comme l'écrit Marc Harteman, «l'école traditionnelle cultivait la pensée, l'école nouvelle cultive la pensée et l'action. L'école mixte est peut-être la première à cultiver réellement le cœur " ${ }^{127}$.

Cependant, pour montrer sa conformité à la déclaration conciliaire, le SGEC insiste sur le fait que la mixité n'est pas incompatible avec le respect de la différence de nature entre les deux sexes. De là, une insistance sur la "vocation" propre à chaque sexe, qui vise aussi à rassurer les traditionalistes. Cette insistance sur la nécessité de respecter la différence des natures masculines et féminines peut expliquer l'absence de lutte contre les stéréotypes masculins et féminins. Mais cette absence était-elle alors particulière à l'enseignement catholique? On remarquera en tout cas qu'aujourd'hui l'enseignement catholique est conscient du problème. Dans un article sur la mixité des sexes publié dans la très officielle revue Documents Épiscopats en 2005, le secrétaire général adjoint de l'enseignement catholique, André Blandin, insiste en effet sur la nécessité de lutter contre les stéréotypes qui favorisent l'orientation des filles vers certaines filières scolaires et vers certains métiers, tout en maintenant une éducation qui respecte la différence homme-femme ${ }^{128}$. Ce point de vue atteste que l'anthropologie catholique n'est pas incompatible avec l'égalité des chances. Le débat porte maintenant sur la notion de gender, récusée par les autorités catholiques ${ }^{129}$.

Yves VERNEUIL Université de Reims Champagne-Ardenne (ESPE) verneuil_yves@yahoo.fr

127 Marc Harteman, art. cit., p. 19.

128 André Blandin, "Le défi de l'altérité ou les mixités à l'école", in "Éduquer, le bonheur de faire grandir", Documents Épiscopat, n 10-11, septembre 2005, fiche 2.

129 Cf. document d'orientation du SGEC sur "L'éducation affective, relationnelle et sexuelle dans les établissements catholiques d'enseignement", 16 avril 2010. 